\title{
Herpesvirus of Turkeys (Meleagridis Herpesvirus 1) Encodes a Functional MicroRNA-221 Homolog with High Sequence Conservation
}

\author{
Yongxiu Yao1, Chan Ding², Venugopal Nair ${ }^{1,3,4^{*}}$ \\ ${ }^{1}$ The Pirbright Institute \& UK-China Centre of Excellence for Research on Avian Diseases, Surrey, UK \\ ${ }^{2}$ Department of Avian Infectious Diseases, Shanghai Veterinary Research Institute, Chinese Academy of Agricultural Sciences, \\ Shanghai, China \\ ${ }^{3}$ The Jenner Institute Laboratories, University of Oxford, Oxford, UK \\ ${ }^{4}$ Department of Zoology, University of Oxford, Oxford, UK \\ Email: *venugopal.nair@pirbright.ac.uk
}

How to cite this paper: Yao, Y.X., Ding, C. and Nair, V. (2019) Herpesvirus of Turkeys (Meleagridis Herpesvirus 1) Encodes a Functional MicroRNA-221 Homolog with High Sequence Conservation. Advances in Microbiology, 9, 728-736.

https://doi.org/10.4236/aim.2019.98044

Received: June 12, 2019

Accepted: August 6, 2019

Published: August 9, 2019

Copyright $\odot 2019$ by author(s) and Scientific Research Publishing Inc. This work is licensed under the Creative Commons Attribution International License (CC BY 4.0).

http://creativecommons.org/licenses/by/4.0/

\begin{abstract}
Herpesviruses account for most of the known virus-encoded miRNAs. Herpesvirus of turkey (HVT), a non-pathogenic avian herpesvirus used as an avian vaccine and viral vector, encodes 28 mature miRNAs. This included HVT-miR-H14-3p that showed almost identical sequence to gga-miR-221, suggesting that it is pirated from the avian host. Although the functional homolog between the two miRNAs has been proposed based on the sequence similarity, the direct experimental evidence is still lacking. In this report, we provide the evidence for the first time that HVT-miR-H14-3p is indeed a gga-miR-221 homolog through modulating the expression of $\mathrm{p} 27^{\mathrm{Kip} 1}$, a known target of miR-221 by binding to its 3'UTR. We also created an HVT-miR-H14-3p deletion virus and show that this miRNA is not essential for in vitro replication.
\end{abstract}

\section{Keywords}

HVT, HVT-miR-H14-3p, Homolog, gga-miR-221, p27 ${ }^{\text {Kip1 }}$

MicroRNAs (miRNAs) are now well-recognized as major regulators of gene expression in a wide spectrum of biological events [1] [2]. They are also used for regulation of gene expression by several viruses, particularly herpesviruses [3] [4]. Unlike many metazoan miRNAs, virus-encoded miRNAs generally show li- 
mited sequence conservation suggesting their independent, and perhaps more rapid, evolution [5]. Despite this, certain virus-encoded miRNAs have evolved to share the "seed" sequences of other miRNAs enabling them to repress common sets of target genes. The best examples of such functional miRNA homologs are the Kaposi's sarcoma-associated herpesvirus-encoded miR-K12-11 [6] [7], the Marek's disease virus (MDV)-encoded miR-M4 [8] and the Simian foamy virus (SFV)-encoded SFVagm-miR-S4-3p [9] all sharing the same 7-nucleotide "seed" sequences of the hsa/gga-miR-155 [10]. KSHV-encoded miR-K12-10a shares seed sequence with the abundant hematopoietic-cell specific host miR-142-3p [11]. Moreover, KSHV-encoded miR-K-3 and its isoform miR-K-3+1 have been shown to be effective mimics of cellular miR-23 [12]. SFVagm-miR-S6-3p, another SFV-encoded miRNA, exhibits seed sequence homology with host immunosuppressive miRNA miR-132 [9]. Similarly, one viral miRNA produced by bovine leukemia virus (BLV), BLV-miR-B4, shares its seed sequence with host B-cell tumorigenic miR-29a [13]. All of these viral miRNA mimics share some of the targets as their host counterparts. Although further validation is required for the proposed orthologs, seed sequence homology has been observed between the murine gammaherpesvirus-68-miR-M1-4 and mmu-miR-151 [4], MDV2-miR-M21 and gga-miR-29 [14] [15].

Herpesvirus of turkey (HVT), known also as Meleagridis herpesvirus 1, is one of the most widely used Marek's disease vaccine and recombinant vaccine vector used against avian diseases. We and others have previously reported the identification of 28 mature miRNAs encoded by HVT [14] [16]. Some of these miRNAs such as HVT-miR-H5, shared the "seed" sequence with MDV1-miR-M9 [17] and MDV2-miR-M28 [15], demonstrating that viruses can encode potentially functional orthologs of both host and virus-encoded miRNAs. However with all these miRNA orthologs, the conservation of the sequences is restricted mostly to the functionally important $6 / 7$ nucleotide seed region, suggestive of a selection pressure to maintain this sequence. Base pairing outside the seed region also influences many miRNA: target interactions although predicting these interactions has remained difficult. A virus-encoded miRNA homolog that retains sequence identity both with the seed and non-seed regions would be valuable for delineating the functions of miRNA regions. HVT-miR-H14-3p [14], also named as HVT-miR6 [18], is the first example of such a miRNA, which shows match in 21/23 nucleotides with the host encoded gga-miR-221 (Figure 1(a)) [14]. Although it has been suggested that novel miRNAs can arise de novo from existing hairpin structures [19], the high degree of sequence homology with gga-miR-221 strongly suggested that HVT-miR-H14-3p was snatched by the virus from the host. Moreover, demonstration of partial sequence conservation between the downstream flanking region of HVT-miR-H14-3p in the HVT genome and the gga-miR-221 locus on chromosome 1 of the chicken genome, supports this conclusion [14]. Since all the other known viral miRNA homologs show conservation only in the seed sequences, it is surprising that HVT-miR-H14-3p 


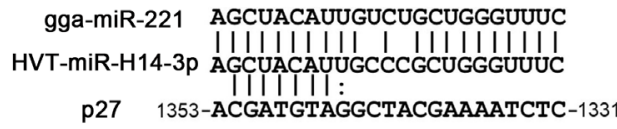

(a)

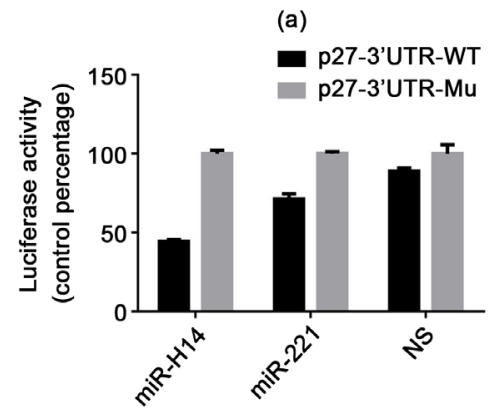

(b)
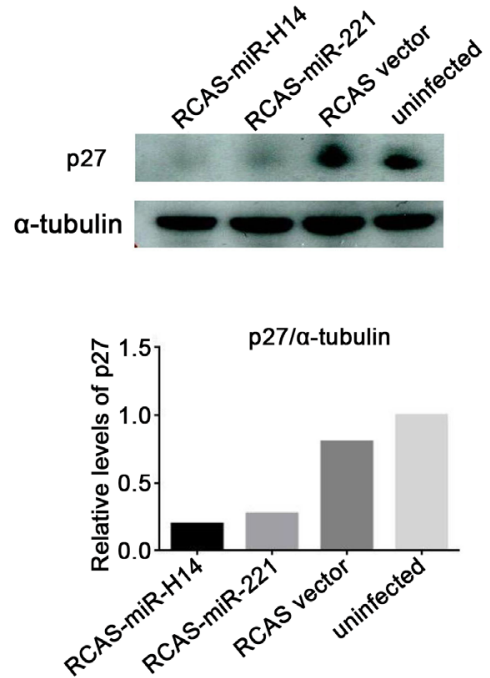

(c)

Figure 1. HVT-miR-H14-3p is a homolog of gga-miR-221. (a) Alignment of mature HVT-miR-H14-3p and gga-miR-221 showing the conservation of sequences along with

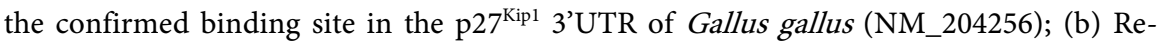
pression of luciferase reporter construct containing the chicken $\mathrm{p} 27^{\mathrm{Kip} 1} 3^{\text {'UTR sequence }}$ inserted downstream of Renilla luciferase in the pSi-CHECK-2 vector. The histogram shows the relative levels of Renilla luciferase in DF-1 cells co-transfected with reporter vectors containing the wild type $(\mathrm{wt})$ or mutated $(\mathrm{mu}) \mathrm{p} 27^{\mathrm{Kip} 1} 3^{\text {'UTR }}$ and gga-miR-221, HVT-miR-H14-3p or non-silencing (NS) expression constructs [27]. The relative expression of Renilla luciferase was determined with the normalized levels of firefly luciferase. For each sample, values from four replicates were used in the analysis. The value from the psiCHECK-2-mutant was set as 1 . Error bars are derived from four replicates; (c) Top panel: Western blot analysis of p27 $7^{\text {Kip } 1}$ expression in RCAS-infected DF-1 cells expressing either HVT-miR-H14-3p or gga-miR-221. Wild type RCAS and uninfected DF-1 cells were used as controls. Levels of $\alpha$-tubulin are shown as loading control. Bottom panel: Relative signal intensities of the p27 Western blot band were quantified using Image Quant and normalized against the corresponding signal from the tubulin band. The signal from un-infected cells was set as 1 .

is maintained almost in its entirety by the virus. This would probably suggest that the non-seed region of HVT-miR-H14-3p also may have a functional role, or there may be structural constraints that require the miRNA to be maintained in its entirety. Alternatively, the acquisition of gga-miR-221 by the virus may only be a recent event, and further virus evolution could lead to emergence of a more conventional miRNA homolog with restricted sequence conservation.

Extensive studies on the cellular miRNAs miR-221/222 have demonstrated their major roles in cell cycle and cancer [20] [21], including their roles in regulating the expression of cell cycle regulatory proteins such as the cyclin-dependent kinase (cdk) inhibitor p $27^{\mathrm{Kip} 1}$ [22]-[27] and $\mathrm{p} 57^{\mathrm{Kip} 2}$ [20] [22] [26]. We have demonstrated that miR-221/222 is expressed at high levels in MDV-transformed chicken T-cell line MSB-1 [17] [28]. We also showed that these miRNAs can modulate the expression of chicken $\mathrm{p} 27^{\mathrm{Kip} 1}$ through the specific sequence in the $3^{\prime} \mathrm{UTR}$ of the chicken $\mathrm{p} 27^{\mathrm{Kip} 1}$ transcript [27]. The functional 
role of these miRNAs in modulating p $27^{\text {Kip1 }}$ protein expression was also confirmed through overexpression studies and by using antagomiRs [27]. On the basis of these studies, we hypothesized that the HVT-miR-H14-3p homolog will have a modulatory role on the expression of the miR-221/222 targets such as the p2 $7^{\text {Kip } 1}$.

To determine if HVT-miR-H14-3p can also target $\mathrm{p} 27^{\text {Kip1}}$, we generated a HVT-miR-H14-3p expression plasmid similar to the miR-221 expression construct reported previously [27], by annealing complementary DNA oligonucleotides HVT-H14-F and HVT-H14-R (Table 1) and cloned into RCAS vector. The ability of HVT-miR-H14-3p to target $\mathrm{p} 27^{\mathrm{Kipl}}$ was first assessed by co-transfection of DF-1 cells with the HVT-miR-H14-3p expression plasmid along with the reporter construct that featured the 3'UTR in its native form (p27-3'UTR-WT) or a mutant construct with three base-pair mutations in each of the two predicted miRNA binding sites (p27-3'UTR-dbMT) fused to the 3'UTR of the renilla luciferase in psiCHECKTM-2 [27]. The miR-221 expression construct and a negative control miRNA (miR-NS) [27] were also included as positive and negative controls respectively. The luciferase expression was assayed 48 hours after transfection using the Dual-Glo Luciferase Assay System (Promega), and the relative expression of renilla luciferase normalised to the levels of firefly luciferase was determined. For each sample, values from four replicates representative of three independent experiments were used in the analysis. Co-transfection of HVT-miR-H14-3p expression vector with p27-3'UTR-WT resulted in 55\% knockdown of renilla luciferase activity, and $30 \%$ reduction was seen by miR-221 expression plasmid (Figure 1(b)). Thus, the reporter assay demonstrated that HVT-miR-H14-3p, similar to miR-221, can target p27 $7^{\text {Kip1 }}$. We next measured the reduction of $\mathrm{p} 27^{\mathrm{Kip} 1}$ protein level by HVT-miR-H14-3p and miR-221 in DF-1 cells to further confirm that HVT-miR-H14-3p and miR-221 are functional orthologs. To maximize the effect of HVT-miR-H14-3p and miR-221 on the target $\mathrm{p} 27^{\mathrm{Kip} 1}$, we used the replication-competent avian retrovirus RCASBP-B-CN-EGFP [27] vector (a generous gift from Dr. Jon Gilthorpe, Kings College London) to generate HVT-miR-H14-3p and miR-221 expression vectors. As replication competent vectors, these constructs allow expression of high levels of miRNAs from the chicken U6 promoter [27] and virus-infected cells can be tracked by the marker EGFP expression. The transfected

Table 1. Sequence of the oligonucleotide primers used (homologous sequences used for recombination are underlined).

\begin{tabular}{cc}
\hline Primers & \multicolumn{1}{c}{ Sequences $\left(5^{\prime}-3^{\prime}\right)$} \\
\hline HVT-H14-F & CGTCATTCAGCGGGCAATGTAGACTGTGTACCAAGTGACAGCTACATTGCCCGCTGGGTTTTTTTTT \\
HVT-H14-R & CGCGAAAAAAAAACCCAGCGGGCAATGTAGCTGTCACTTGGTACACAGTCTACATTGCCCGCTGAATGA \\
HVT-H14-KD-F & GCTAAGCAGTTAGTTGTACGGAAATGATGACAGATCAGACTAATGCACGGAGTGTAGGCTGGAGCTGCTTC \\
HVT-H14-KD-R & CACATATGGCACAAAAATCGCGGGCGGTCTACACTCTTGCATTTCCAGACAGCCATTCCGGGGATCCGTCGAC \\
HVT-miR-galK-F & GCTAAGCAGTTAGTTGTACGGAAATGATGACAGATCAGACTAATGCACGGACTGTTGACAATTAATCATCGGCA \\
HVT-miR-galK-R & CACATATGGCACAAAAATCGCGGGCGGTCTACACTCTTGCATTTCCAGACAGCCTCAGCACTGTCCTGCTCCTT \\
\hline
\end{tabular}


DF-1 cells were passed a few times to allow the spread of the virus, and analysed for $\mathrm{p} 27^{\mathrm{Kip} 1}$ expression by Western blotting. The expression of HVT-miR-H14-3p and miR-221 significantly reduced the $\mathrm{p} 27^{\mathrm{Kip} 1}$ protein expression in these cells compared to the wild type RCAS-infected or uninfected DF-1 cells (Figure $1(\mathrm{c})$ ), showing that $\mathrm{p} 27^{\mathrm{Kip} 1}$ is a target for both HVT-miR-H14-3p and miR-221.

Having demonstrated that miR-221 homolog is functional in modulating the expression of at least one of the target protein, p2 $7^{\text {Kip1 }}$, we wanted to examine whether it is either essential for replication or can provide any functional advantages for the virus. For this, we generated HVT-miR-H14-3p deletion mutants of HVT by mutagenesis of pHVT3 [29] using standard procedures [30] [31] in SW105 strain of E. coli (kindly provided by Dr. N. Copeland, NCI Frederick, MD). The two copies of the HVT-miR-H14-3p were deleted sequentially in two steps, first by inserting $\mathrm{Kan}^{\mathrm{R}}$ cassette amplified by PCR using primers HVT-H14-KD-F and HVT-H14-KD-R. After flipping out the $\mathrm{Kan}^{\mathrm{R}}$ cassette, the second copy was deleted by the insertion of a ga/K cassette amplified by the primers HVT-miR-galK-F and HVT-miR-galK-R. The construct from which both copies of the HVT-miR-H14-3p deleted was named as pHVT3-H14-00. The ga/K cassette in the construct was also used as a negative selection marker for restoration of HVT-miR-H14-3p in the revertant construct pHVT3-H14-R0. The accuracy of the deletions was checked by PCR using oligonucleotide primers (Table 1). Viruses were reconstituted in chicken embryo fibroblast cultures (CEF) transfected with the BAC DNA constructs. Detection of virus plaques in cells transfected with each of the constructs showed that deletion of the HVT-miR-H14-3p did not affect the reconstitution of the virus. In order to examine whether the deletion of HVT-miR-H14-3p affected virus replication, in vitro growth kinetics of the PHVT3 virus was compared with that of the mutant viruses in a time course plaque assay. The 3 viruses showed similar replication kinetics (Figure 2(a)) demonstrating that HVT-miR-H14-3p did not affect the in vitro virus replication.

We also evaluated the expression of HVT-miR-H14-3p by Northern blot analysis in CEF infected with the viruses. These results confirmed that while both pHVT3 and the pHVT3-H14-R0 viruses expressed HVT-miR-H14-3p, the deletion of both copies abolished its expression (Figure 2(b)). We also examined the effect of abolishing the expression of HVT-miR-H14-3p on the levels of $\mathrm{p} 27^{\mathrm{kip} 1}$ at three days post infection in CEF. Western blotting analysis of $\mathrm{p} 27^{\mathrm{kip} 1}$ in CEF infected with different viruses showed that the levels p2 $7^{\mathrm{kip} 1}$ were almost identical regardless of the expression of HVT-miR-H14-3p (Figure 2(c)). It is not clear why the levels of p $27^{\text {kip1 }}$ were not affected by HVT-miR-H14-3p in infected CEF. Since we have demonstrated that the $\mathrm{p} 27^{\mathrm{kip} 1}$ expression can be significantly reduced by overexpression of HVT-miR-H14-3p using RCAS vector in DF-1 cells (Figure 1(c)), it is most likely related to the differences in the levels of infection due to the cell associated nature of HVT as well as in the expression levels of the miRNA and target proteins in these cell types. On the other hand, the 


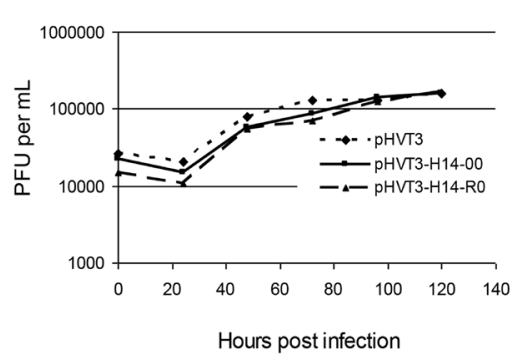

(a)

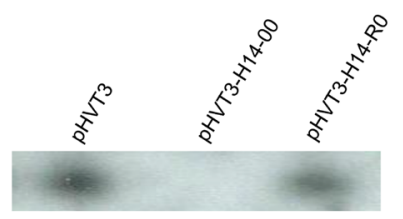

(b)
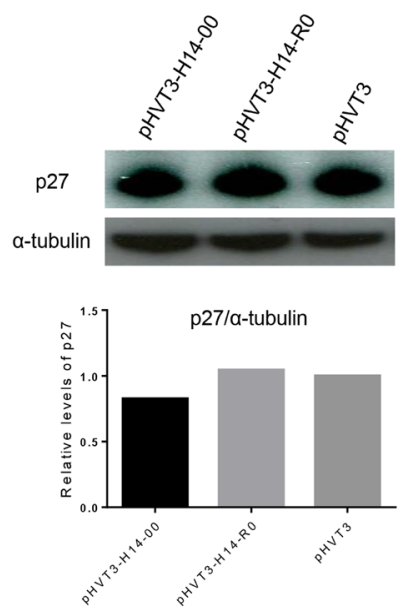

(c)

Figure 2. Characterization of HVT-miR-H14-3p mutant viruses. (a) Comparison of the in vitro growth of HVT in infected CEF. Virus titres expressed as plaque numbers were determined at different times after inoculation with pHVT3, pHVT3-H14-00 and pHVT3-HVT14-R0 viruses on CEF monolayers; (b) Northern blot analysis of RNA extracted from virus-infected CEF to examine the HVT-miR-H14-3p expression; (c) Top panel: Western blot of HVT-infected CEF for the detection of p27 ${ }^{\text {Kip1 }}$. Levels of $\alpha$-tubulin are shown as loading control. Bottom panel: Relative signal intensities of the $\mathrm{p} 27^{\mathrm{Kip} 1}$ Western blot band were quantified using Image Quant and normalized against the corresponding signal from the tubulin band. The signal from pHVT3-infected cells was set as 1.

dynamics of miRNA-mediated regulation of target transcripts is very complex with a single miRNA capable of silencing multiple targets, as well as a single transcript being regulated by multiple miRNAs [32]. Thus the absence of any visible effects on the $\mathrm{p} 27^{\mathrm{kip} 1}$ levels or on the replication of HVT in infected cells regardless of HVT-miR-H14-3p expression would suggest the complexity of miRNA-mediated gene regulation, where the existing compensating and complementing pathways may mask the effect of a single miRNA. Although we did not see an effect in vitro, the HVT replication dynamics in other cell types is poorly understood; it is possible that in certain cell types, particularly when the endogenous gga-miR-221 levels are limited, the function of this viral homolog may be important.

\section{Acknowledgements}

This project was supported by the Biotechnology and Biological Sciences Research Council (BBSRC) grants BBS/E/I/00007032, BB/R007896/1 and BBSRC Newton Fund Joint Centre Awards on "UK-China Centre of Excellence for Research on Avian Diseases".

\section{Conflicts of Interest}

The authors report no conflict of interest. 


\section{References}

[1] Hwang, H.W. and Mendell, J.T. (2006) Micrornas in Cell Proliferation, Cell Death, and Tumorigenesis. British Journal of Cancer, 94, 776-780.

https://doi.org/10.1038/sj.bjc.6603023

[2] Fu, L.L., Wen, X., Bao, J.K. and Liu, B. (2012) Microrna-Modulated Autophagic Signaling Networks in Cancer. The international Journal of Biochemistry \& Cell Biology, 44, 733-736. https://doi.org/10.1016/j.biocel.2012.02.004

[3] Cullen, B.R. (2009) Viral and Cellular Messenger RNA Targets of Viral Micrornas. Nature, 457, 421-425. https://doi.org/10.1038/nature07757

[4] Gottwein, E. and Cullen, B.R. (2008) Viral and Cellular Micrornas as Determinants of Viral Pathogenesis and Immunity. Cell Host \& Microbe, 3, 375-387. https://doi.org/10.1016/j.chom.2008.05.002

[5] Nair, V. and Zavolan, M. (2006) Virus-Encoded Micrornas: Novel Regulators of Gene Expression. Trends in Microbiology, 14, 169-175. https://doi.org/10.1016/j.tim.2006.02.007

[6] Gottwein, E., Mukherjee, N., Sachse, C., Frenzel, C., Majoros, W.H., Chi, J.T., Braich, R., Manoharan, M., Soutschek, J., Ohler, U., et al. (2007) A Viral Microrna Functions as an Orthologue of Cellular Mir-155. Nature, 450, 1096-1099. https://doi.org/10.1038/nature05992

[7] Skalsky, R.L., Samols, M.A., Plaisance, K.B., Boss, I.W., Riva, A., Lopez, M.C., Baker, H.V. and Renne, R. (2007) Kaposi's Sarcoma-Associated Herpesvirus Encodes an Ortholog of Mir-155. Journal of Virology, 81, 12836-12845. https://doi.org/10.1128/JVI.01804-07

[8] Zhao, Y., Yao, Y., Xu, H., Lambeth, L., Smith, L.P., Kgosana, L., Wang, X. and Nair, V. (2009) A Functional Microrna-155 Ortholog Encoded by the Oncogenic Marek's Disease Virus. Journal of Virology, 83, 489-492. https://doi.org/10.1128/JVI.01166-08

[9] Kincaid, R.P., Chen, Y., Cox, J.E., Rethwilm, A. and Sullivan, C.S. (2014) Noncanonical Microrna (Mirna) Biogenesis Gives Rise to Retroviral Mimics of Lymphoproliferative and Immunosuppressive Host Mirnas. mBio, 5, e00074. https://doi.org/10.1128/mBio.00074-14

[10] Faraoni, I., Antonetti, F.R., Cardone, J. and Bonmassar, E. (2009) Mir-155 Gene: A Typical Multifunctional Microrna. Biochimica et Biophysica Acta, 1792, 497-505. https://doi.org/10.1016/j.bbadis.2009.02.013

[11] Gottwein, E., Corcoran, D.L., Mukherjee, N., Skalsky, R.L., Hafner, M., Nusbaum, J.D., Shamulailatpam, P., Love, C.L., Dave, S.S., Tuschl, T., et al. (2011) Viral Microrna Targetome of Kshv-Infected Primary Effusion Lymphoma Cell Lines. Cell Host \& Microbe, 10, 515-526. https://doi.org/10.1016/j.chom.2011.09.012

[12] Manzano, M., Shamulailatpam, P., Raja, A.N. and Gottwein, E. (2013) Kaposi's Sarcoma-Associated Herpesvirus Encodes a Mimic of Cellular Mir-23. Journal of Virology, 87, 11821-11830. https://doi.org/10.1128/JVI.01692-13

[13] Kincaid, R.P., Burke, J.M. and Sullivan, C.S. (2012) RNA Virus Microrna That Mimics a b-Cell Oncomir. Proceedings of the National Academy of Sciences of the United States of America, 109, 3077-3082. https://doi.org/10.1073/pnas.1116107109

[14] Waidner, L.A., Morgan, R.W., Anderson, A.S., Bernberg, E.L., Kamboj, S., Garcia, M., Riblet, S.M., Ouyang, M., Isaacs, G.K., Markis, M., et al. (2009) Micrornas of Gallid and Meleagrid Herpesviruses Show Generally Conserved Genomic Locations and Are Virus-Specific. Virology, 388, 128-136.

https://doi.org/10.1016/j.virol.2009.02.043 
[15] Yao, Y., Zhao, Y., Xu, H., Smith, L.P., Lawrie, C.H., Sewer, A., Zavolan, M. and Nair, V. (2007) Marek's Disease Virus Type 2 (Mdv-2)-Encoded Micrornas Show No Sequence Conservation with Those Encoded by Mdv-1. Journal of Virology, 81, 7164-7170. https://doi.org/10.1128/JVI.00112-07

[16] Yao, Y., Zhao, Y., Smith, L.P., Watson, M. and Nair, V. (2009) Novel Micrornas Encoded by Herpesvirus of Turkeys (hvt): Evidence of Mirna Evolution by Duplication. Journal of Virology, 83, 6969-6973. https://doi.org/10.1128/JVI.00322-09

[17] Yao, Y., Zhao, Y., Xu, H., Smith, L.P., Lawrie, C.H., Watson, M. and Nair, V. (2008) Microrna Profile of Marek's Disease Virus-Transformed t-Cell Line Msb-1: Predominance of Virus-Encoded Micrornas. Journal of Virology, 82, 4007-4015. https://doi.org/10.1128/JVI.02659-07

[18] Yao, Y., Zhao, Y., Smith, L.P., Watson, M. and Nair, V. (2009) Novel Micrornas (Mirnas) Encoded by Herpesvirus of Turkeys: Evidence of Mirna Evolution by Duplication. Journal of Virology, 83, 6969-6973.

https://doi.org/10.1128/JVI.00322-09

[19] Liu, N., Okamura, K., Tyler, D.M., Phillips, M.D., Chung, W.J. and Lai, E.C. (2008) The Evolution and Functional Diversification of Animal Microrna Genes. Cell Research, 18, 985-996. https://doi.org/10.1038/cr.2008.278

[20] Medina, R., Zaidi, S.K., Liu, C.G., Stein, J.L., van Wijnen, A.J., Croce, C.M. and Stein, G.S. (2008) Micrornas 221 and 222 Bypass Quiescence and Compromise Cell Survival. Cancer Research, 68, 2773-2780. https://doi.org/10.1158/0008-5472.CAN-07-6754

[21] Song, J., Ouyang, Y., Che, J., Li, X., Zhao, Y., Yang, K., Zhao, X., Chen, Y., Fan, C. and Yuan, W. (2017) Potential Value of Mir-221/222 as Diagnostic, Prognostic, and Therapeutic Biomarkers for Diseases. Frontiers in Immunology, 8, 56. https://doi.org/10.3389/fimmu.2017.00056

[22] Fornari, F., Gramantieri, L., Ferracin, M., Veronese, A., Sabbioni, S., Calin, G.A., Grazi, G.L., Giovannini, C., Croce, C.M., Bolondi, L., et al. (2008) Mir-221 Controls cdkn1c/p57 and cdkn1b/p27 Expression in Human Hepatocellular Carcinoma. Oncogene, 27, 5651-5661. https://doi.org/10.1038/onc.2008.178

[23] Galardi, S., Mercatelli, N., Giorda, E., Massalini, S., Frajese, G.V., Ciafre, S.A. and Farace, M.G. (2007) Mir-221 and Mir-222 Expression Affects the Proliferation Potential of Human Prostate Carcinoma Cell Lines by Targeting p27kip1. The Journal of Biological Chemistry, 282, 23716-23724. https://doi.org/10.1074/jbc.M701805200

[24] Le Sage, C., Nagel, R., Egan, D.A., Schrier, M., Mesman, E., Mangiola, A., Anile, C., Maira, G., Mercatelli, N., Ciafre, S.A., et al. (2007) Regulation of the p27(kip1) Tumor Suppressor by Mir-221 and Mir-222 Promotes Cancer Cell Proliferation. The EMBO Journal, 26, 3699-3708. https://doi.org/10.1038/sj.emboj.7601790

[25] Visone, R., Russo, L., Pallante, P., De Martino, I., Ferraro, A., Leone, V., Borbone, E., Petrocca, F., Alder, H., Croce, C.M., et al. (2007) Micrornas (Mir)-221 and Mir-222, Both Overexpressed in Human Thyroid Papillary Carcinomas, Regulate p27kip1 Protein Levels and Cell Cycle. Endocrine-Related Cancer, 14, 791-798. https://doi.org/10.1677/ERC-07-0129

[26] Liu, X., Cheng, Y., Zhang, S., Lin, Y., Yang, J. and Zhang, C. (2009) A Necessary Role of Mir-221 and Mir-222 in Vascular Smooth Muscle Cell Proliferation and Neointimal Hyperplasia. Circulation Research, 104, 476-487. https://doi.org/10.1161/CIRCRESAHA.108.185363

[27] Lambeth, L.S., Yao, Y., Smith, L.P., Zhao, Y. and Nair, V.K. (2009) Micrornas 221 and 222 Target p27kip1 in Marek's Disease Virus-Transformed Tumour Cell Line Msb-1. Journal of General Virology, 90, 1164-1171. 
https://doi.org/10.1099/vir.0.007831-0

[28] Yao, Y., Zhao, Y., Smith, L.P., Lawrie, C.H., Saunders, N.J., Watson, M. and Nair, V. (2009) Differential Expression of Micrornas in Marek's Disease Virus-Transformed t-Lymphoma Cell Lines. Journal of General Virology, 90, 1551-1559. https://doi.org/10.1099/vir.0.009902-0

[29] Baigent, S.J., Petherbridge, L.J., Smith, L.P., Zhao, Y., Chesters, P.M. and Nair, V.K. (2006) Herpesvirus of Turkey Reconstituted from Bacterial Artificial Chromosome Clones Induces Protection against Marek's Disease. Journal of General Virology, 87, 769-776. https://doi.org/10.1099/vir.0.81498-0

[30] Warming, S., Costantino, N., Court, D.L., Jenkins, N.A. and Copeland, N.G. (2005) Simple and Highly Efficient BAC Recombineering Using Galk Selection. Nucleic Acids Research, 33, e36. https://doi.org/10.1093/nar/gni035

[31] Yu, D., Ellis, H.M., Lee, E.C., Jenkins, N.A., Copeland, N.G. and Court, D.L. (2000) An Efficient Recombination System for Chromosome Engineering in Escherichia coli. Proceedings of the National Academy of Sciences of the United States of America, 97, 5978-5983. https://doi.org/10.1073/pnas.100127597

[32] Bartel, D.P. (2009) Micrornas: Target Recognition and Regulatory Functions. Cell, 136, 215-233. https://doi.org/10.1016/j.cell.2009.01.002 